Progress in Flight Physics 5 (2013) 139-152

DOI: $10.1051 /$ eucass/201305139

(C) Owned by the authors, published by EDP Sciences, 2013

\title{
FLOWFIELDS AROUND SUPERSONIC AERODYNAMIC BODIES UNDER THE ACTION OF ASYMMETRIC ENERGY RELEASE
}

O. A. Azarova ${ }^{1}$, D. Knight ${ }^{2}$, and Yu. F. Kolesnichenko ${ }^{3}$

\author{
${ }^{1}$ Dorodnicyn Computing Centre \\ Russian Academy of Sciences \\ 40 Vavilova Str., Moscow 119333, Russia \\ ${ }^{2}$ Rutgers University \\ 98 Brett Road, Piscataway, New Brunswick, NJ 08854, USA \\ ${ }^{3}$ Joint Institute for High Temperatures \\ Russian Academy of Sciences \\ 13-2 Izhorskaya Str., Moscow 125412, Russia
}

\begin{abstract}
The interaction of an infinite microwave filament and a shock layer is analyzed numerically on the basis of the Euler system of equations. The filament is regarded as a heated rarefied channel (heat layer). Flow details for asymmetrical filament location are researched including the formation of a new position of the stagnation point and the dependence of the front drag force on the filament characteristics and location. The origin of a lift/pitch force in the case of zero angle of attack is discussed. This force is shown to be a function of the shift value from the symmetry axis of the heat layer and the degree of the gas rarefaction in it. The mechanism of the lift/pitch force origination is revealed. These phenomena are analyzed for blunt and pointed bodies at freestream Mach number 1.89 and a wide class of values of infinite filament characteristics: the rarefaction factor and the disposition relative to the body.
\end{abstract}

\section{INTRODUCTION}

The effect of heat inhomogeneities on a flow reconstruction over an aerodynamic body in a supersonic flow has been investigated since the end of the last century (see the survey in [1] and additional surveys in $[2,3]$ ). In $[4,5]$, the possibility of the supersonic flow control by a space-distributed energy supply was pointed

This is an Open Access article distributed under the terms of the Creative Commons Attribution License 2.0, which permits unrestricted use, distribution, and reproduction in any medium, provided the original work is properly cited. 
out. Flow structure reorganization caused by the interaction of the heated rarefied channel (or the heated layer) with a shock layer was analyzed in [6]. In [7], the application of this phenomenon for modeling of the microwave energy effect on aerodynamic characteristics of bodies for the purpose of the flow control improvement was considered. The results of the computation of the asymmetrically located (relative to a streamlined body) energy release in a supersonic flow were discussed in $[8,9]$, including the generation and dynamics of the lift force under zero angle of attack. Steady flow structures for considered flow modes were obtained and researched in [10]. In this paper, flows over blunt and pointed bodies are analyzed for an asymmetric filament location relative to the body. The dynamics of drag and lift/pitch forces is discussed. The flow modes characterized by the generation of steady periodic structures are also considered.

\section{STATEMENT OF THE PROBLEM}

The interaction of a microwave filament with a planar shock layer is numerically analyzed on the basis of the system of Euler equations for an ideal gas in two dimensions:

$$
\frac{\partial \mathbf{U}}{\partial t}+\frac{\partial \mathbf{F}}{\partial x}+\frac{\partial \mathbf{G}}{\partial y}=0
$$

Here,

$$
\mathbf{U}=\left(\begin{array}{c}
\rho \\
\rho u \\
\rho v \\
E_{S}
\end{array}\right) ; \quad \mathbf{F}=\left(\begin{array}{c}
\rho u \\
p+\rho u^{2} \\
\rho u v \\
u\left(E_{S}+p\right)
\end{array}\right) ; \quad \mathbf{G}=\left(\begin{array}{c}
\rho v \\
\rho u v \\
p+\rho v^{2} \\
v\left(E_{S}+p\right)
\end{array}\right)
$$

where $\rho$ and $p$ are the density and the pressure of the gas; $u$ and $v$ are the $x$ and $y$-components of the gas velocity, $E_{S}=\rho\left(\varepsilon+0.5\left(u^{2}+v^{2}\right)\right)$, and the units of measure are: $l_{n}=10^{-1} \mathrm{~m}, t_{n}=1.6 \cdot 10^{-4} \mathrm{~s}, u_{n}=6.27 \cdot 10^{2} \mathrm{~m} / \mathrm{s}$. The statement of the problem is analogous to the authors' previous work in [7]. The Mach number of the oncoming flow was 1.89. On the entrance boundary the parameters of the oncoming flow are used. Reflection (slip) boundary conditions are utilized on the body surface. A no-reflection boundary condition is applied on the exit boundary. The energy deposition by the microwave pulse is modeled as the instantaneous creation of a heated infinite rarefied channel ("filament"). The filament enters the computational domain at the entrance boundary $(x=0)$ as a channel of low density $\rho_{i}, \rho_{i}=\alpha_{\rho} \rho_{0}$ for $0 \leq r \leq 0.5 d$. The static pressure and the velocity of the channel are equal to those of the undisturbed flow. Thus, the channel is regarded as a heated layer. Nondimensional undisturbed flow parameters corresponding to the normal conditions are: $\rho_{0}=1, p_{0}=0.2, u_{0}=1$, and $v_{0}=0$. The filament is assumed to arise instantly in the steady flow in front of the bow shock wave at the time moment $t_{i}$. Space and time filament characteristics are 


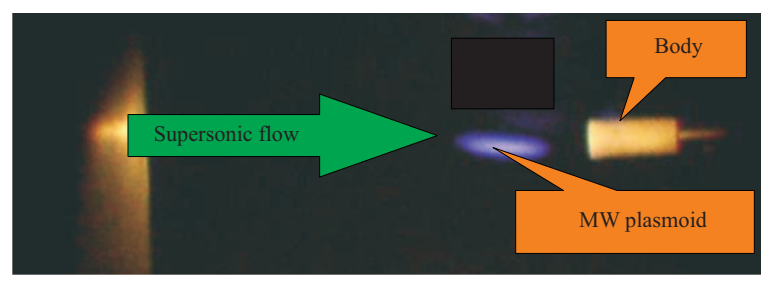

Figure 1 Microwave filament (experiment).

in agreement with the parameters of the heated areas obtained experimentally as a result of microwave energy injection into the air [7]. A slightly modified difference scheme from [11] is used. A photograph of the experimental filament image and a schematic sketch for the statement of the computations are presented in Figs. 1 and 2. The considered flow parameters are presented in Table 1.

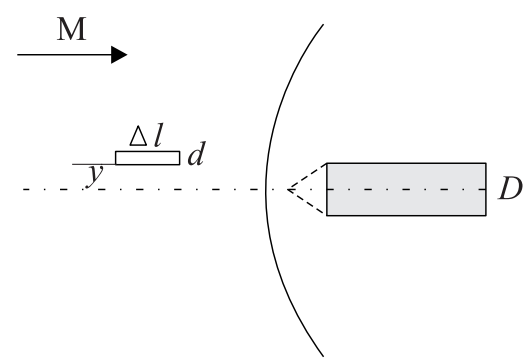

Figure 2 Schematic flow configuration

Table 1 Governing dimensionless parameters

\begin{tabular}{|c|c|c|c|}
\hline Type & Description & Definition & Value \\
\hline \multirow{4}{*}{ Flow } & Mach number & $\mathrm{M}$ & 1.89 \\
\hline & Specific heats ratio & $\gamma$ & 1.4 \\
\hline & Body's diameter & $\bar{D}$ & 0.2 \\
\hline & $\begin{array}{l}\text { Body's shape } \\
(\beta-\text { half top angle })\end{array}$ & & $\begin{array}{l}\text { blunt, } \\
\text { pointed }(\operatorname{tg} \beta=1.0 ; \operatorname{tg} \beta=1 / 3)\end{array}$ \\
\hline \multirow{4}{*}{ Filament } & $\begin{array}{l}\text { Degree of gas rarefaction } \\
\text { (density ratio) }\end{array}$ & $\alpha_{\rho}$ & $0.4-0.6$ \\
\hline & Diameter & $d / D$ & $0.127,0.254$ \\
\hline & Length (duration) & $\Delta l / D$ & $\infty$ \\
\hline & $\begin{array}{l}\text { Location } \\
\text { ( } y \text { - the distance between } \\
\text { the lower filament } \\
\text { boundary and the axis } \\
\text { of symmetry) }\end{array}$ & $y / D$ & $\begin{array}{l}-0.13 \text { (symmetric), } \\
0.08,0.16,0.24,0.4 \\
\text { (asymmetric) }\end{array}$ \\
\hline
\end{tabular}




\section{RESULTS}

\subsection{Specific Features of Flows in the Case of Asymmetric Filament Location}

Figures 3 and 4 present the steady-flow fields of the interaction of the energy release located symmetrically and asymmetrically towards the blunt body. In this part, $d / D=0.254$. Under the symmetric disposition of an energy source channel in the steady state, a triangle heated area in front of a body is formed (see Fig. 3). The warm stream from the heated source enters the triangle area and is divided in two equal parts at some distance from the body. Each part of the stream is then convected down along the appropriate lateral body's surface. The stagnation point is disposed in the center of the front body's surface just as in the case of a flow without channel, the pressure in this point being sufficiently less under the channel action causing the face drag force reduction. For asymmetric filament location (if it is placed up from the axis of symmetry), the stagnation point forms in the lower position than that for the case of the symmetric location (see Fig. 4). In this point, the pressure value exceeds that for the flow without the filament by tens of percent. The contact discontinuities form a new triangle structure, the below contact discontinuity turns near the body and becomes parallel to the body's front surface. An additional shock wave with respect to the symmetrical filament location is generated from the left boundary of the rarefaction wave with the center in the bottom body's corner (see Figs. 3 and 4a). For more rarefied gas in the filament, this shock wave does not appear (see Fig. 4, right column); the threshold value of the rarefaction degree $\alpha_{\rho}$ is between 0.5 and 0.6.

In Fig. 5, the averaged dependencies of the relative values of the stagnation pressure, the drag force and $y$-coordinate of the stagnation point on the body

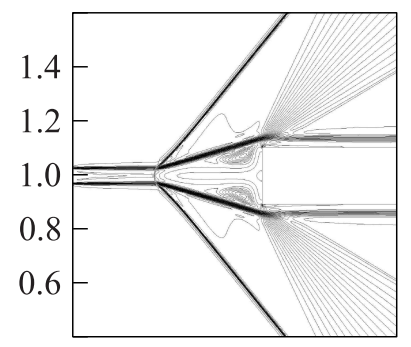

(a)

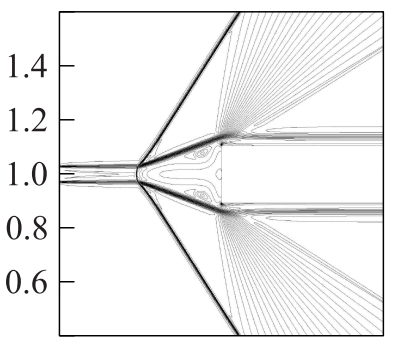

(b)

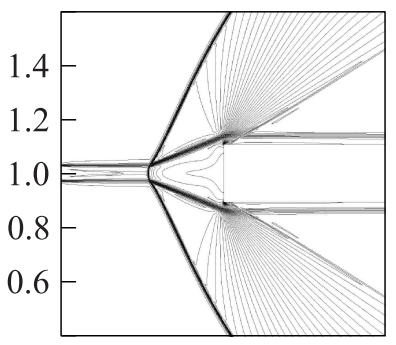

(c)

Figure 3 Steady flowfields of the interaction of symmetrically located filament with shock layer from blunt body (density, isochors) $(y / D=-0.13):(a) \alpha_{\rho}=0.4 ;(b) 0.5$; and $(b) \alpha_{\rho}=0.6$ 

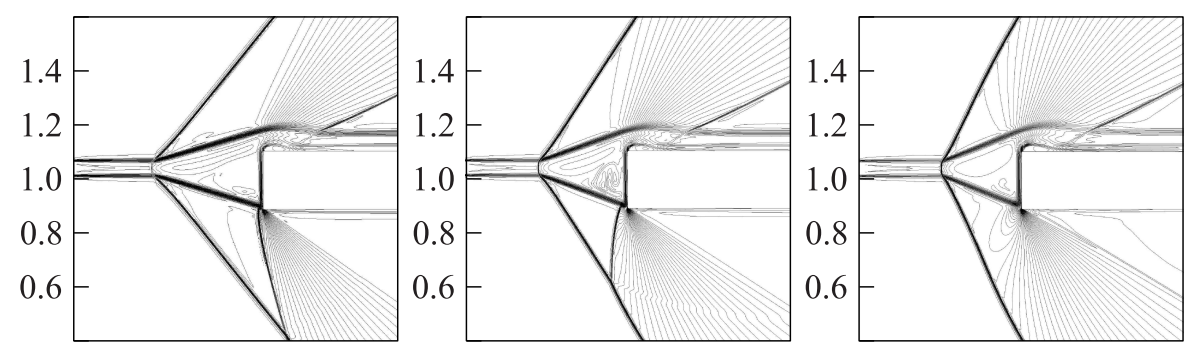

(a)
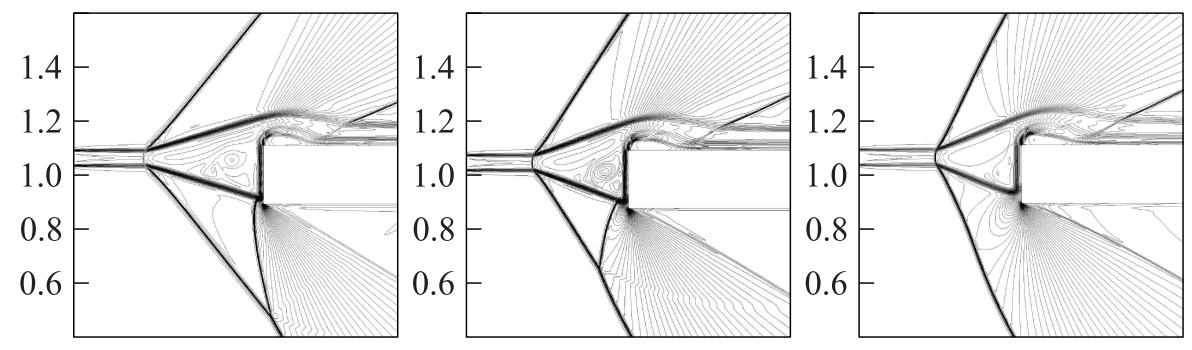

(b)
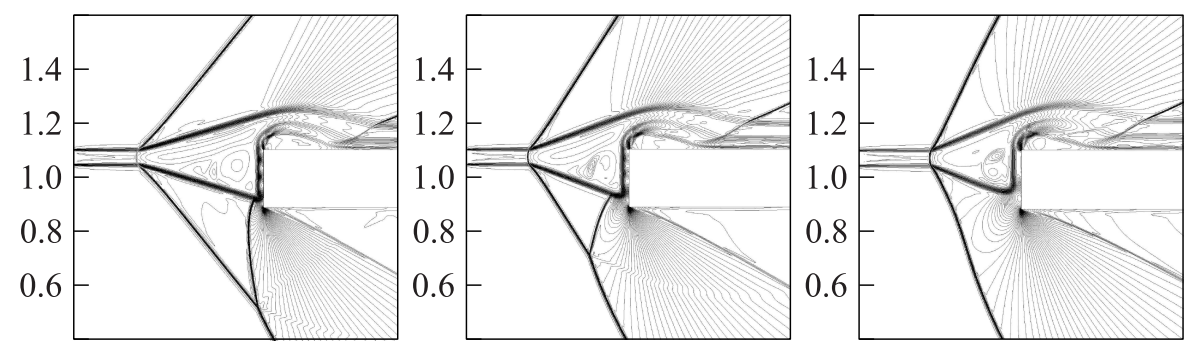

(c)

Figure 4 Steady flowfields of the interaction of asymmetrically located filament with shock layer from blunt body (density, isochors): (a) $y / D=0.08 ;(b) 0.16$; and (c) $y / D=0.24$. Left column refers to $\alpha_{\rho}=0.4$; middle column to $\alpha_{\rho}=0.5$; and right column to $\alpha_{\rho}=0.6$

over the filament location are presented for different rarefaction degree in the filament. Here, $p_{s}, F$, and $y_{s}$ are the stagnation pressure, the drag force, and the stagnation point coordinate for the flow with filament, respectively, and $p_{s}^{0}$, $F^{0}$, and $y_{s}^{0}$ are the similar parameters for the flow without filament.

A face drag force reduction is observed in all cases. A pair vortex structure generated at the first stage of the energy release/shock layer interaction was shown to be the reason for the primary drag force reduction [7]. The stagnation 


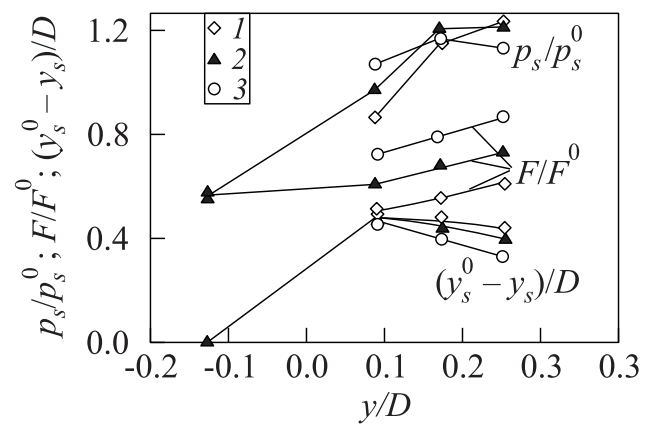

Figure 5 Dependencies of relative values of stagnation pressure, drag force and $r$-coordinate of the stagnation point on the body over the filament location for different rarefaction degree in the filament : $1-\alpha_{\rho}=0.4 ; 2-0.5$; and $3-\alpha_{\rho}=0.6$

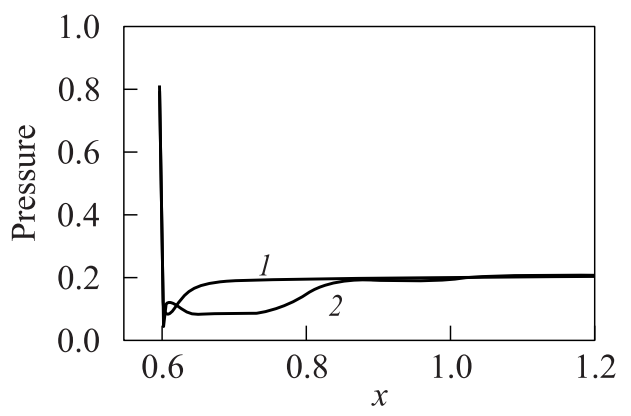

Figure 6 Pressure on the lower (1) and upper (2) lateral body's surfaces

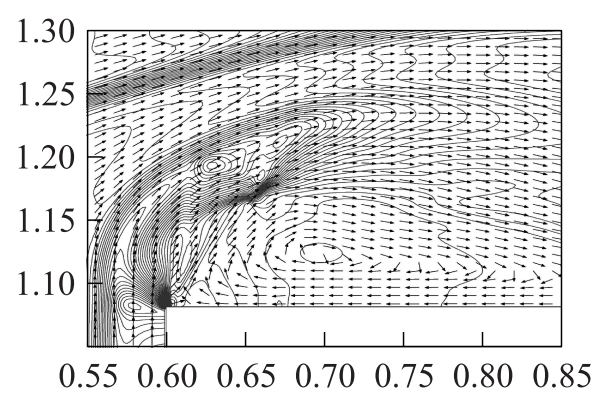

Figure 7 Velocity and density (isochors) fields inside the upper separation area, $y / D=0.4$ and $\alpha_{\rho}=0.5$ 


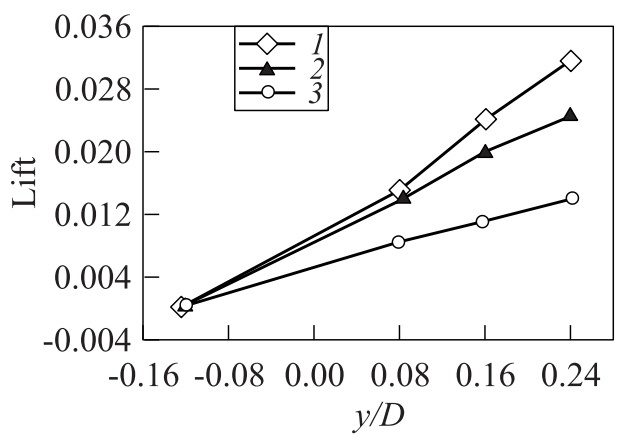

Figure 8 Dependence of the lift force over $y / D$ for different values of $\alpha_{\rho}: 1-0.4$; $2-0.5 ;$ and $3-0.6$

point location on the front of the body is lower and the drag force reduction is greater for greater values of $y / D$. These dependencies are linear over the value of the relative distance from the filament to the axis of symmetry $y / D$.

Moving the filament up from the axis leads to the overall up shift of the heated triangle area, and, as a consequence, to opening of a lower part of the body to the action of a cold supersonic stream (causing a new stagnation point position). The most part of the stream goes down along the lower lateral surface. The rest (small) part of this cold flow goes up along the front surface of the body pressing the warm triangle structure away from this surface. Reaching the upper corner of the body, this cold flow comes into contact with the warm flow exiting from the triangle area, turns to the right and then is convected down along the upper lateral surface, leading to the origination of a separation area. In Fig. 6, the distributions of the pressure along the lateral body surfaces are presented. It is seen that at the lower surface, the pressure quickly approaches the static pressure of the oncoming flow (note that the dimensionless freestream pressure value is $p_{0}=0.2$ ). On the contrary, at the upper surface, it achieves this value only at some distance downstream the separation area. Inside this area, the flow is of a vortex structure (Fig. 7) and the pressure value is less than the freestream pressure. As a result of this pressure difference, a net vertical force arises which could be distinguished as a lift force if it were not limited in the spatial domain of its application. It is more correct to describe it as a pitch force. The lift/pitch force is a function of a shift value from the symmetry axis of the thermal layer and the degree of rarefaction in it (Fig. 8). It is seen that within the limits of investigated parameters, this force rises linearly with the shift distance of the heated channel from the axis and is approximately inversely proportional to the degree of rarefaction in the channel.

Let compare stationary flowfields for different shapes of the body (for $\alpha_{\rho}$ $=0.4$ and 0.6 ): the streamlining of blunt body (case 1, Fig. $9 a$ ) and two cases of 


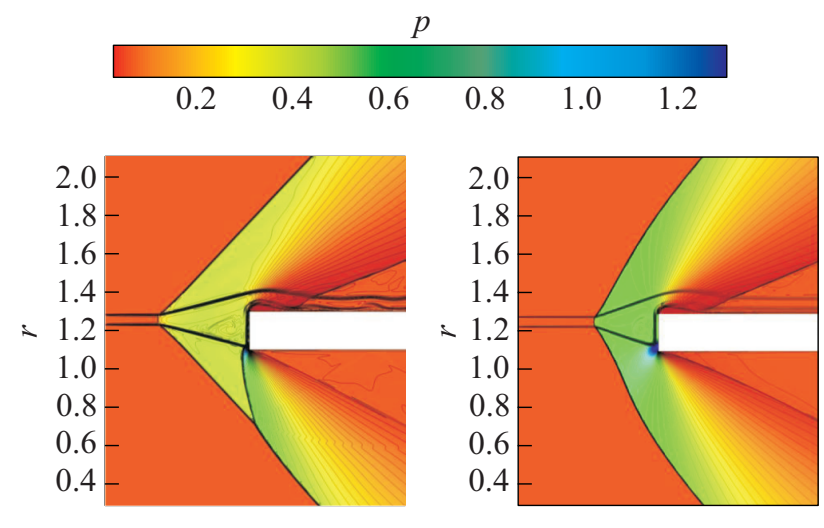

(a)
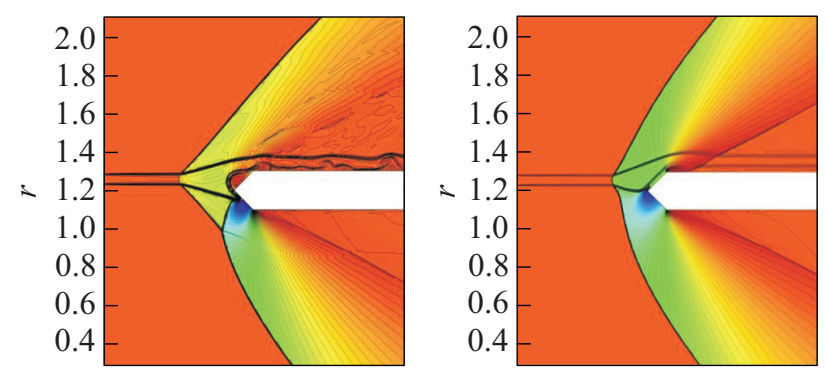

(b)
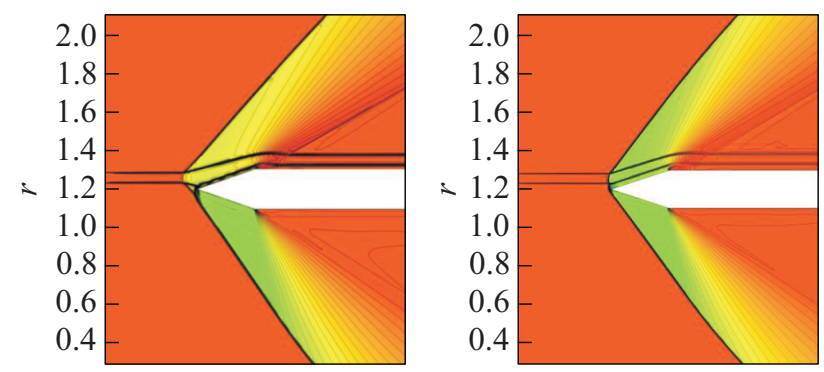

(c)

Figure 9 Steady flowfields of the interaction of asymmetrically located filament with shock layer, left column refers to $\alpha_{\rho}=0.4$, and right column to $\alpha_{\rho}=0.6$ (density, isochors, and pressure, colors): (a) blunt body; (b) $\operatorname{tg} \beta=1.0$; and (c) $\operatorname{tg} \beta=1 / 3$. 


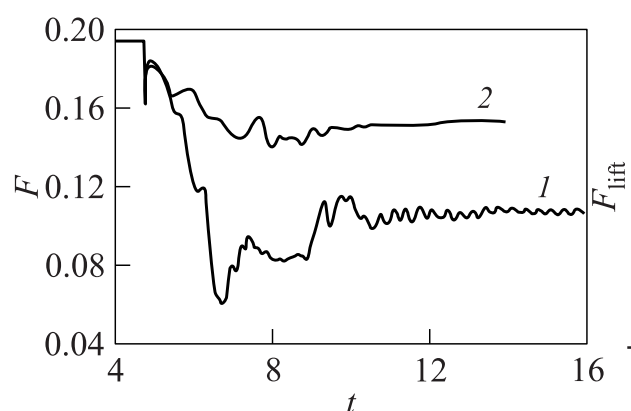

(a)

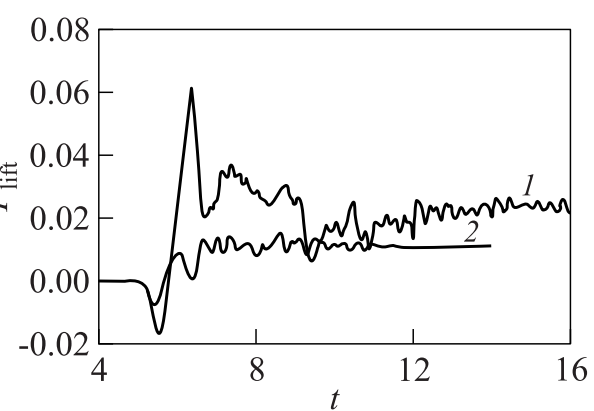

(b)

Figure 10 Dynamics of defining flow parameters, blunt body $\left(1-\alpha_{\rho}=0.4\right.$, and $\left.2-\alpha_{\rho}=0.6\right):(a)$ face drag forces; and (b) lift forces

streamlining pointed bodies with a wedge as a front shape: $\operatorname{tg} \beta=1.0$ (case 2 , Fig. $9 b$ ) and $\operatorname{tg} \beta=1 / 3$ (case 3, Fig. $9 c$ ). At Mach number 1.89, in the first two cases, the initial detached bow shock wave regime (without an energy supply) is realized; in the last, the initial flow with an attached shock occurs. Here, $y=0.16 D$. It is seen that the flow modes for different rarefaction degree are characterized by different shock structures near the front part of the bodies. The interaction with the filament shifted to $y=0.16 D$ above the axis of symmetry leads to a drag force reduction and the development of a lift/pitch force in spite of the zero angle of attack (Figs. 10-12). Here,

$$
F=\int_{y_{b}}^{y_{b}+D} p d y ; \quad F_{\text {lift }}=F_{n}-F_{v}
$$

where $y_{b}$ is the lower body's $y$-coordinate, $F_{n}$ and $F_{v}$ are the drag forces of the lower and upper body's surfaces. The dynamics of drag and lift forces for blunt body (case 1) is presented in Fig. 10 and for the cases 2 and 3 of pointed body in Figs. 11 and 12. In Figs. $11 a$ and $12 a, F_{k_{n}}$ and $F_{k_{v}}$ are the front drag force components for the bottom and top surfaces of the pointed part of the aerodynamic body, so $F=F_{k_{n}}+F_{k_{v}}$. It can be seen that the drag force is reduced in all the cases examined - at $45.0 \%, 31.8 \%$, and $8.3 \%$, respectively, and the drag force reduction is greater for smaller $\alpha_{\rho}$. It should also be noticed that the bow shock wave is weaker inside the heated rarefied channel in all considered cases. This weakness (calculated on the basis of the density drop on the shock front) is about $11.8 \%$.

As for lift (pitch) forces for all the cases (see Figs. 10b, 11b, and 12b) some peculiarities are observed between the three cases. Using the blunt body as a reference, one may see a redistribution of the pressure along the lateral surfaces, 


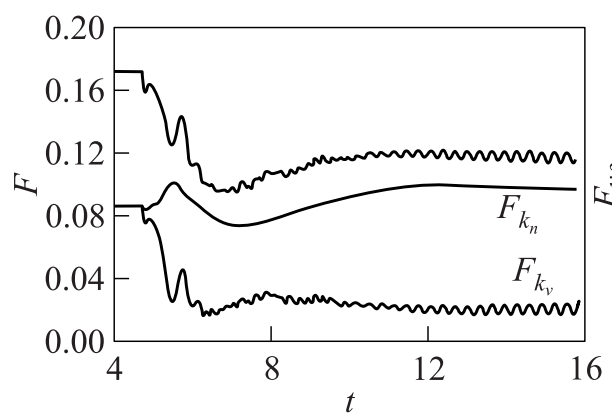

(a)

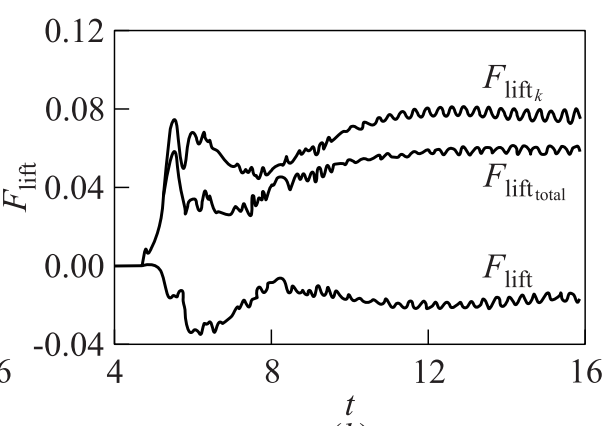

(b)

Figure 11 Dynamics of defining flow parameters, pointed body, $\operatorname{tg} \beta=1.0$ (the case of primary detached bow shock), $\alpha_{\rho}=0.4$ : ( $a$ ) face drag forces; and (b) lift forces

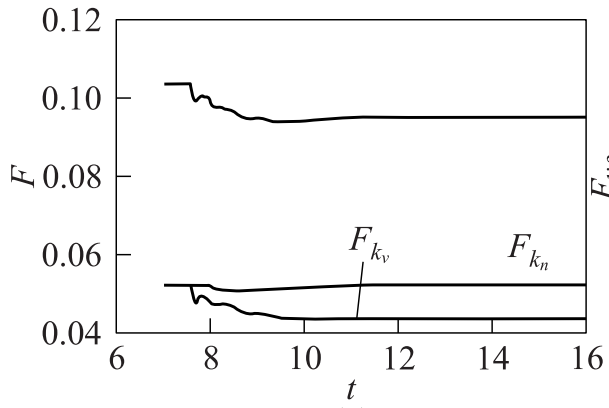

(a)

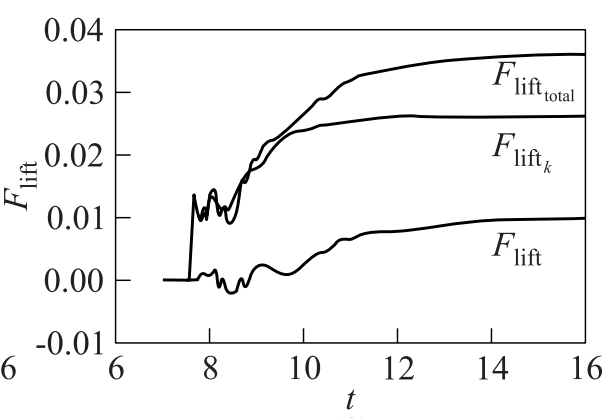

(b)

Figure 12 Dynamics of defining flow parameters, pointed body, $\operatorname{tg} \beta=1 / 3$ (the case of primary attached bow shock), $\alpha_{\rho}=0.4$ : ( $a$ ) face drag forces; and $(b)$ lift forces

which is especially visible for the second body shape (see Fig. $11 b$ ). Here, $F_{\text {lift }}$ and $F_{\text {lift }_{k}}$ are the lift forces formed by the upper and lower lateral surfaces and the lift force formed by upper and lower surfaces of the pointed part of the aerodynamic body and $F_{\text {lift total }_{\text {th }}}=F_{\text {lift }}+F_{\text {lift }_{k}}$. In this case the separation area is formed on the pointed part of the body. However the lateral force on the upper surface turns out to become greater than on the lower one, so it decreases the lift force from the front (pointed) part of the body $F_{\text {lift }_{k}}$. In the third case, the separation area is not formed, the lateral force on the upper surface is again less than on the lower one and this gives a positive contribution to the net lift force (see Fig. 12b). Nevertheless, in this case the lift force $F_{\text {lift }}$ is twice as less than for the blunt body.

The comparison of the drag and lift forces mean values in the steady state for the cases 1-3 for different values of the rarefaction degree in the filament is 
Table 2 Characteristics of the drag and lift forces

\begin{tabular}{|c|c|c|c|c|c|c|}
\hline$\alpha_{\rho}$ & $\frac{F^{0}-F}{F^{0}}$ & $\frac{F_{k_{n}}^{0}-F_{k_{n}}}{F_{k_{n}}^{0}}$ & $\frac{F_{k_{v}}^{0}-F_{k_{v}}}{F_{k_{v}}^{0}}$ & $\frac{F_{\text {lift }_{\text {total }}}}{F_{n}^{0}+F_{k}^{0}}$ & $\frac{F_{\text {lift }}}{F_{n}^{0}}$ & $\frac{F_{\text {lift }_{k}}}{F_{k}^{0}}$ \\
\hline \multicolumn{7}{|c|}{ Blunt body (case 1$)$} \\
\hline 0.4 & $45.0 \%$ & - & - & $19.4 \%$ & $19.4 \%$ & - \\
\hline 0.6 & $21.2 \%$ & - & - & $9.1 \%$ & $9.1 \%$ & - \\
\hline \multicolumn{7}{|c|}{ Pointed body, $\operatorname{tg} \beta=1.0$ (case 2 ) } \\
\hline 0.4 & $31.8 \%$ & $-12.3 \%$ & $76.0 \%$ & $26.9 \%$ & $-12.2 \%$ & $88.4 \%$ \\
\hline 0.6 & $11.2 \%$ & $-8.2 \%$ & $30.6 \%$ & $12.1 \%$ & $-5.0 \%$ & $38.9 \%$ \\
\hline \multicolumn{7}{|c|}{ Pointed body, $\operatorname{tg} \beta=1 / 3$ (case 3 ) } \\
\hline$\overline{0.4}$ & $8.3 \%$ & $-0.2 \%$ & $16.8 \%$ & $11.3 \%$ & $6.0 \%$ & $17.0 \%$ \\
\hline 0.6 & $-0.4 \%$ & $0.1 \%$ & $-0.8 \%$ & $1.1 \%$ & $2.9 \%$ & $-0.9 \%$ \\
\hline
\end{tabular}

presented in Table 2. Here, the parameters are related to the according values in the absence of the filament $F_{k_{n}}^{0}, F_{k_{v}}^{0}, F_{n}^{0}$, and $F_{k}^{0}$ (the last force is formed by the lower surface of the pointed part).

For the blunt body, the values of drag force reduction and the values of lift forces differ approximately twice for $\alpha_{\rho}=0.4$ and 0.6. Note that for $\alpha_{\rho}=0.4$, the flow mode with the bottom shock wave takes place and for $\alpha_{\rho}=0.6$, the flow mode without this shock wave takes place. In case 2 , the values of the drag forces reduction differ in three times and the values of lift forces differ more than in two times. In case 3, the drag force reduction is still registered (8.3\%) for $\alpha_{\rho}=0.4$ and is not registered for $\alpha_{\rho}=0.6$, the values of total lift forces differ in ten times. Thus, the effects are stronger for smaller $\alpha_{\rho}$ and with increasing sharpness of an aerodynamic body, the flow modes with the bottom shock waves become more preferable for the drag force reduction and the total lift force obtaining, the most input in these forces being provided by the front pointed parts of the bodies.

\subsection{Steady Flow Modes with Periodical Flowfield Structures Generation}

In the case of an asymmetric filament location relative to the body, a specific type of flowfield was observed near the body's face. For the particular set of inflow parameters, steady-flow structures with some oscillations of the parameters in their structure elements are observed in the area of a cooler gas between the vertical contact discontinuity and the front surface of the body. Fields of density (in isochors) and velocity in the steady flow structures with two $(y / D=0.24)$ and three $(y / D=0.2)$ elements are presented accordingly in Figs. $13 a$ and 13b. Depending on the freestream parameters (including rarefied heated channel 


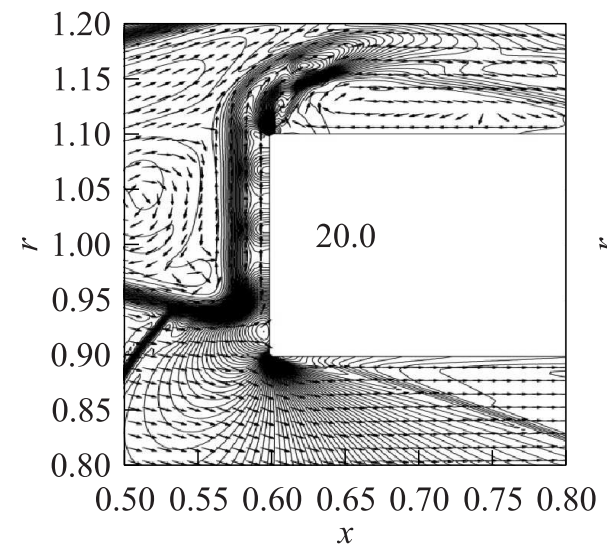

(a)

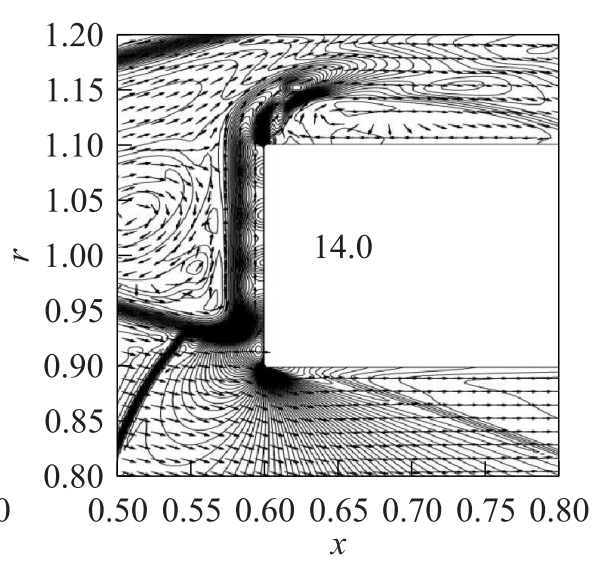

(b)

Figure 13 Density (isochors) and velocity in the periodical steady-flow structures with two $(a)$ and three $(b)$ elements; $\mathrm{M}=1.89$ and $\alpha_{\rho}=0.5[10]$

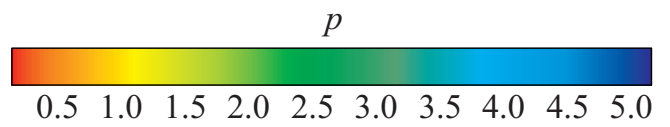

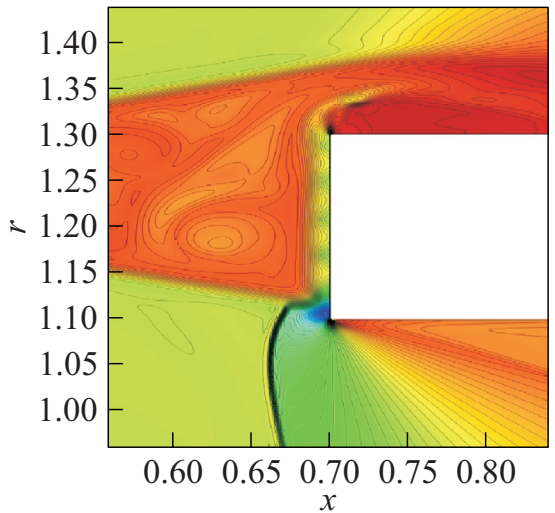

(a)

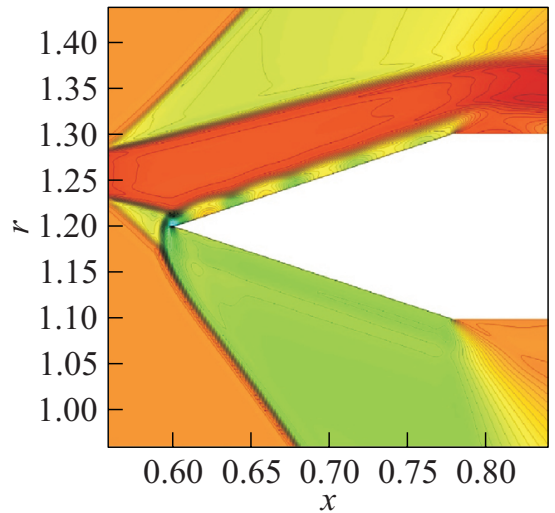

(b)

Figure 14 Density (isochors and colors) in the periodical steady-flow structures with four elements, $\mathrm{M}=1.89, \alpha_{\rho}=0.4, y / D=0.16$ : (a) blunt body, $d / D=0.127$; and (b) pointed body, $d / D=0.254$. 
parameters) the number of elements in these steady structures varies from one to four. The transverse velocity component generation during the evolution of the structures shows the possibility of the presence of a tangential shock instability under these conditions. The mechanism for the generation of these structures is connected with the multiple reflection of a primary compression wave inside the flow between the tangential shock and the wall [10]. This mechanism is similar to that of the generation and forming structures which accompany the MilesRibner instability of super-reflection observed experimentally (in a cylinder flow) in a shallow water [12].

Figure 14 demonstrates steady flow modes with the periodical structures of four elements generated in the cases of blunt (Fig. 14a) and pointed (Fig. 14b) bodies (the structure's characteristics turned out to be weakly dependent on the filament radius). It is seen that for the same heated channel location relative to the body $(y / D=0.16)$ and the same rarefaction degree $\left(\alpha_{\rho}=0.4\right)$ of the gas in the filament, the structures in the case of blunt and pointed bodies contain the same number of the elements (four).

\section{REFERENCES}

1. Knight, D. 2008. Survey of aerodynamic drag reduction at high speed by energy deposition. J. Propul. Power 24(6):1153-67.

2. Zheltovodov, A. 2002. Development of the studies on energy deposition for application to the problems of supersonic aerodynamics. Preprint No. 10-2002. Novosibirsk, Russia: Khristianovich Institute of Theoretical and Applied Mechanics.

3. Fomin, V., P. Tretyakov, and J.-P. Taran. 2004. Flow control using various plasma and aerodynamic approaches (short review). Aerospace Sci. Technol. 8(5):411-21.

4. Georgievsky, P. Y., and V. A. Levin. 1988. Supersonic flow over bodies in the presence of external energy input. Lett. J. Techn. Phys. 14(8):684-87.

5. Georgievsky, P. Y., and V. A. Levin. 2002. Effective control of supersonic flows over different bodies by an energy input to ambient Flow. 4th Workshop on MagnetoPlasma Aerodynamics in Aerospace Applications Proceedings. Ed. V. A. Bityurin. Moscow: IVTAN. 40-43.

6. Artem'ev, V. I., V. I. Bergel'son, I. V. Nemchinov, T. I. Orlova, V. A. Smirnov, and V. M. Hazins. 1989. Changing the regime of supersonic streamlining obstacle via arising the thin channel of low density. Mech. Fluids Gases 5:146-51.

7. Kolesnichenko, Yu.F., V.G. Brovkin, O.A. Azarova, V.G. Grudnitsky, V. A. Lashkov, and I. Ch. Mashek. 2002. Microwave energy release regimes for drag reduction in supersonic flows. AIAA Paper No. 2002-0353:1-13.

8. Nemchinov, I. V., V. I. Artem'ev, V. I. Bergel'son, V. M. Hazins, T. I. Orlova, and V.A. Rybakov. 1994. Rearrangement of the bow shock shape using a "Hot Spike." Shock Waves 4:35-40. 
9. Knight, D., O. A. Azarova, and Yu.F. Kolesnichenko. 2009. On details of flow control via characteristics and location of microwave filament during its interaction with supersonic blunt body. AIAA Paper No. 2009-847:1-21.

10. Azarova, O. A. 2010. Numerical experiments on modeling of steady structures in supersonic flows with asymmetrical energy supply, J. Comput. Math. Math. Phys. 50(10):1840-53.

11. Belotserkovsky, O. M., V. G. Grudnitsky, and Yu. A. Prohorchuk. 1983. Difference scheme of the second order of precision on a minimal stencil for hyperbolic equations. J. Comput. Math. Math. Phys. 23(1):119-26.

12. Fridman, A. M. 2008. Prediction and discovery of strongest hydrodynamic instabilities caused by velocity shock: Theory and experiment. Adv. Phys. Sci. 178(3): 225-42. 